\title{
2018 Proceedings
}

Stitched Together: Community Engagement for Undergraduate Student Learning in Apparel Design

Chanjuan Chen, Kendra Lapolla and Archana Mehta Kent State University, USA

Keywords: Community, engagement, upcycling, local

The purpose of this research is to understand the impact of a community engagement approach for both undergraduate students and a nonprofit organization supporting refugee women in the creation of sustainable fashion products to be locally made and sold. As defined by The Carnegie Classification (2018), "Community engagement describes collaboration between institutions of higher education and their larger communities for the mutually beneficial exchange of knowledge and resources in a context of partnership and reciprocity." For this approach, students design upcycled products with donated fabrics and garments from the nonprofit organization and share their skills with the local community. The organization and local refugee women are then able to learn construction techniques that enable them to create local fashion products, culminating in an approach where all participating members mutually benefit through the exchange of design, construction knowledge, and resources. This collaboration also promotes sustainability by creating products locally with secondhand garments to meet the needs of the community which "cut transportation of goods, create jobs near markets and enable closer control of environmental standards (Fletcher \& Gerose, 2012)."

Upon receiving IRB approval, the community engagement project was introduced to college juniors and seniors in a sustainable apparel design studio course. The project lasted a total of three weeks and fourteen students participated. Twelve women from the local nonprofit organization were invited to collaborate with the students to create upcycled products to exhibit and sell to the public on a later date. This nonprofit organization supports refugee women by offering bi-weekly sewing classes at a local community center and aims to provide them with community resources, increase self-confidence and boost their earning capacity.

The students and the refugee women were assigned into five groups by the researchers with two to three students and women in each group. In the first week of the project, students did market research, sketching and sampling to come up with garment or accessory designs that were made from donated denim jeans and saree garments based on the skill sets of the women and the local culture and needs of products. Students also prepared a demonstration plan for their designs. On the second week, the students and the women met in a local community center where the organization provides their bi-weekly workshops. During the $2 \frac{1}{2} 2$ hour workshop, students taught the women in their groups how to construct the garments or accessories. Students communicated their product ideas to the women with the aid of visuals such as pictures or notes in a step-by-step process. At the end of the workshop, the women not only learned how to create the specific product from the students, they also had the chance to pick their fabrics and design the surface of the products with students' help. In the last week of the project, student groups

Page 1 of 3

( ) 2018, International Textile and Apparel Association, Inc. ALL RIGHTS RESERVED ITAA Proceedings, \#75 - http://itaaonline.org 
worked on the branding for the products by creating tags and logos while the women prepared to sell the products on the organization's website and during local events.

Once the project was completed, students who agreed to participate in this study were asked about their learning experience in a qualitative open-ended survey. Responses from twelve of the fourteen students were received and analyzed for learning outcomes and effectiveness of the project. All students responded positively to the project and appreciated collaborating with the women from the community. Many commented that this was a new experience to design an upcycled product and teach others to construct them. For example, one student said "I learned there are so many ways to upcycle and make new beautiful things and also how I can benefit others in the community from my design background." Another commented, "I learned that upcycling is pretty difficult, but helped us understand how important design can really contribute to creating something someone else really wants to make and sell." Working in a group with other students also instilled the value of teamwork and promoted clear communication. For instance, one student said, "Group work is essential in the industry so this was good practice." Another student said, "Working with other students gave me a different insight." Many students have also appreciated the importance of visually communicating how to manufacture their designs. For example, one student commented, "My favorite part was sewing the demo and creating the presentation to be easy to understand," and "If I did it again, I would simplify the group's design so that it is easier for the women." Finally, students had a few suggestions to improve the project design including: 1) change the date of the workshop to a class meeting time since it was hosted on a Saturday morning for convenience to the women from the community, and 2) make the workshop longer in order to finish a couple more complicated designs.

In order to more fully understand the impact of community engagement approaches, women who took the workshop were invited to participate in a focus group interview to share their experiences. Seven of the twelve women participated in the interview with audio recording used by the researchers. Overall the women responded positively to the workshop and were grateful to learn new skills to better prepare them for future employment. One woman said, "It helped us to be independent." Another commented, "I like what I made, it's a lovely bag and it will be a good thing to sell." When asked about working with the students, the women commented the students were friendly and easy to work with. A few women expressed that by working with students from the college, it gave them hope for the future.

Community-based learning engages students by using their own community as the source and focus of learning and gives them opportunities to apply new skills and practice them in novel settings (Melaville, Berg, \& Blank, 2006). The community engagement project provided the students the opportunity to design upcycled products that are locally made and sold while working with community members outside the university to enhance their design, construction and communication skills. Additionally, by collaborating with the students, the community members were able to learn sewing and design skills for potential job opportunities. The findings pertaining to learning outcomes and attitudes regarding the experience from both students and community members will contribute to future plans in generating additional course projects that incorporate both sustainability and community engagement approaches.

Page 2 of 3

(C) 2018, International Textile and Apparel Association, Inc. ALL RIGHTS RESERVED ITAA Proceedings, \#75 - http://itaaonline.org 
Fletcher, K \& Gerose, L. (2012). Fashion \& Sustainability: Design for Change. London: Laurence King Publishing Ltd.

Melaville, A. I., Berg, A. C., \& Blank, M. J. (2006). Community-based learning: Engaging students for success and citizenship. Washington, D.C.: Coalition for Community Schools.

The Carnegie Classification of Institutions of Higher Education ${ }^{\circledR}$. (2018). Retrieved March 28, 2018, from http://carnegieclassifications.iu.edu/ 